\title{
Effective inhibition of hepatitis B virus replication by small interfering RNAs expressed from human foamy virus vectors
}

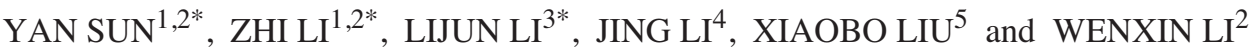 \\ ${ }^{1}$ College of Life Sciences, Shaanxi Normal University, Xi'an, Shaanxi 710062; ${ }^{2}$ State Key Laboratory of Virology, \\ College of Life Sciences, Wuhan University, Wuhan, Hubei 430072; ${ }^{3}$ Bioengineering Institute, Jimei University, \\ Xiamen, Fujian 361021; ${ }^{4}$ Department of Infection Disease, Xiangfan Central Hospital, Xiangfan, \\ Hubei 441021; ${ }^{5}$ Wuhan Aimin Pharmaceutical Co., Ltd, Wuhan, Hubei 430010, P.R. China
}

Received October 27, 2006; Accepted December 11, 2006

\begin{abstract}
RNA interference (RNAi) mediated by doublestranded small interfering RNA (siRNA) is a novel mechanism of sequence-specific, post-transcriptional gene silencing. There has been much research into the use of RNAi for the treatment of human diseases. Many viruses, including hepatitis B virus (HBV), are susceptible to inhibition by this mechanism. However, for RNAi to be efficacious therapeutically, effective RNAi targeting sequences and a suitable delivery system are required. In this study, we employed a polymerase chain reaction (PCR)-based siRNA expression strategy to rapidly screen for effective siRNA sequences. Two effective siRNAs sequences (designated as S2 and X1) which reduced the HBV RNA by $>90 \%$ were identified. For delivering the siRNAs, they were cloned into a human foamy virus (HFV)-based vector to generate single siRNA expression vectors HFVU6-siS2, HFVU6-siX1 and a dual siRNA expression vector HFVU6-siSX. The results showed that these siRNA vectors effectively inhibited multiple HBV gene expression and viral DNA replication based on ELISA and quantitative PCR analysis. HFVU6siSX which simultaneously expressed two siRNAs that targeted the $\mathrm{S}$ and $\mathrm{X}$ genes of $\mathrm{HBV}$ is the most potent inhibitor of HBV replication. In addition, the repression of HBV RNA and DNA was stable for up to 3 months posttransduction as determined by RT-PCR and Southern blotting. Collectively, the PCR-based siRNA expression
\end{abstract}

Correspondence to: Dr Wenxin Li, State Key Laboratory of Virology, College of Life Science, Wuhan University, Wuhan 430072, P.R. China

Dr Zhi Li, College of Life Sciences, Shaanxi Normal University, Xi'an, Shaanxi 710062, P.R. China

E-mail: lizhi@snnu.edu.cn

${ }^{*}$ Contributed equally

Key words: hepatitis B virus, RNA interference, small interfering RNA, antiviral, human foamy virus strategy provides a rapid and easy approach for testing candidate anti-HBV siRNA sequences and for cloning selected siRNA expression cassettes into a vector. RNAi based on the HFV vector was able to achieve effective, longterm inhibition of HBV gene expression and viral DNA replication. The combination of the two techniques may provide a powerful tool in the treatment of viral infection.

\section{Introduction}

Hepatitis B virus (HBV) infection is one of the major threats to public health. It is estimated that, worldwide, approximately 2 billion people have been infected and about 350 million have chronic HBV infection even though effective vaccines are available (1-4). These carriers are at high risk for developing $\mathrm{HBV}$-associated diseases, including chronic hepatitis, liver cirrhosis and primary hepatocellular carcinoma. Worldwide, more than 1 million carriers die from these diseases each year (1). To date, interferon and nucleoside analogue combinational treatment has had only limited success. Thus new therapeutic approaches are urgently needed.

RNA interference (RNAi) is a natural process in eukaryotic cells by which double-stranded RNA initiates and directs sequence-specific, post-transcriptional silencing of homologous genes. It is believed to have evolved as a host defense mechanism directed at transposable elements and infecting viruses $(5,6)$. Recently, RNAi has been applied to inhibit the replication of several pathogenic human viruses. HBV appears to be an attractive target of RNAi, as its pregenome (pg) functions as both mRNA and replication template in the viral life cycle, all of its transcripts have common 3 ' ends, and multiple viral RNAs can be inhibited by a single short interfering RNA (siRNA). Therefore, RNAi is well suited for developing novel treatments for HBV infection.

Successful application of RNAi in the treatment of HBV infection primarily depends on two important factors. One is to screen specific RNAi targeting sequences which could effectively knockdown HBV transcripts. Konishi et al and McCaffrey et al have demonstrated that siRNAs targeted to different sequences of the HBV genome varied in their overall inhibiting effect, and only a limited number of siRNAs were capable of inducing highly effective inhibition $(7,8)$. In 
our study, a PCR-based siRNA expression strategy was employed to rapidly screen for effective siRNA sequences. Briefly, the siRNA expression constructs containing the U6 promoter and short hairpin DNA were established by overlap PCR, and the PCR products were directly transfected into cells resulting in functional expression of siRNAs. The other factor is to develop an efficient intracellular delivery system for siRNAs or the vectors expressing the corresponding siRNAs. Methods relying on lipid-based transfection reagents to introduce synthesized siRNAs or vectors into cells are either inefficient or unsuitable for use in animals. Viral vectors are good candidates for transducing siRNAs, and here a novel HFV vector was utilized to construct siRNA expression vectors. The HFV vector offers the advantages of efficiently infecting the cells and producing stable gene expression in the infected cells. In this study, we report the effects of HFV vector-directed siRNAs on HBV gene expression and replication.

\section{Materials and methods}

Targeting sites of siRNA on HBV genome. From the complete genome of HBV (GenBank Accession no. U95551), nine regions were selected as the targeted sequences using siRNA target finder program of Ambion (http://www.ambion.com/ techlib/misc/siRNA_finder.html). The selected sequences were subjected to global BLAST to ensure that they did not have significant sequence homology with human genome sequences. These sequences targeting the position of the core region of 230,268 , and 332; S region of 69,302 , and 609 ; and $X$ region of 271,283 , and 306 were as follows (Fig. 1A): C1, 5'-AAGATCCAGCATCTAGAGACC-3'; C2, 5'-AAGT TCAGGCAACTCTTGTGG-3'; C3, 5'-AAGAGAAACCGT TATAGAGTA-3'; S1, 5'-AAGAATCCTCACAATACCGC A-3'; S2, 5'-AAGGTATGTTGCCCGTTTGTC-3'; S3, 5'-AA GTCTGTACAGCATCTTGAG-3'; X1, 5'-AAGGTCTTAC ATAAGAGGACT-3'; X2, 5'-AAGAGGACTCTTGGACT CTCT-3'; and X3, 5'-AATGTCAACGACCGACCTTGA-3'. Based on the previous report, a random siRNA (5'-GCGCG CTTTGTAGGATTCG-3') was selected as negative control siRNA (NC) (7).

Preparation of PCR products containing U6 promoter and shDNA. Human U6 promoter was amplified using the plasmid pAVU6+27 (9) as a template with the pair of primers: $\mathrm{P}_{\mathrm{U} 6} \mathrm{~F}, 5$ '-CATATCCGGAAAGGTCGGGCAGGAA

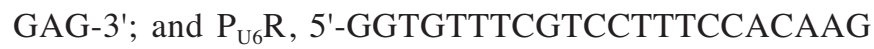
ATATAT-3'. Primer $\mathrm{P}_{\mathrm{U} 6} \mathrm{~F}$ contains the AccIII restriction site underlined in the sequence, which enables directional cloning of PCR products into viral vectors.

Human U6 promoter followed by a 19-nt sense strand of siRNA, a 9-nt loop, a 19-nt anti-sense strand of siRNA, and a stretch of six deoxythymidines were amplified by two-step overlap PCR, as previously described $(10,11)$. In the first round of PCR, the reverse primer (3' primer 1) consisting of $\left(5^{\prime}\right)$ the 9-nt loop complementary sequence (5'-TCTCTTGA A-3'), a 19-nt anti-sense sequence and a complementary sequence to the $11 \mathrm{nt}$ at the $3^{\prime}$-end of the human U6 promoter (3') was used. The first PCR reaction mixture (1 $\mu 1)$ was reamplified in the second round of the PCR reaction using
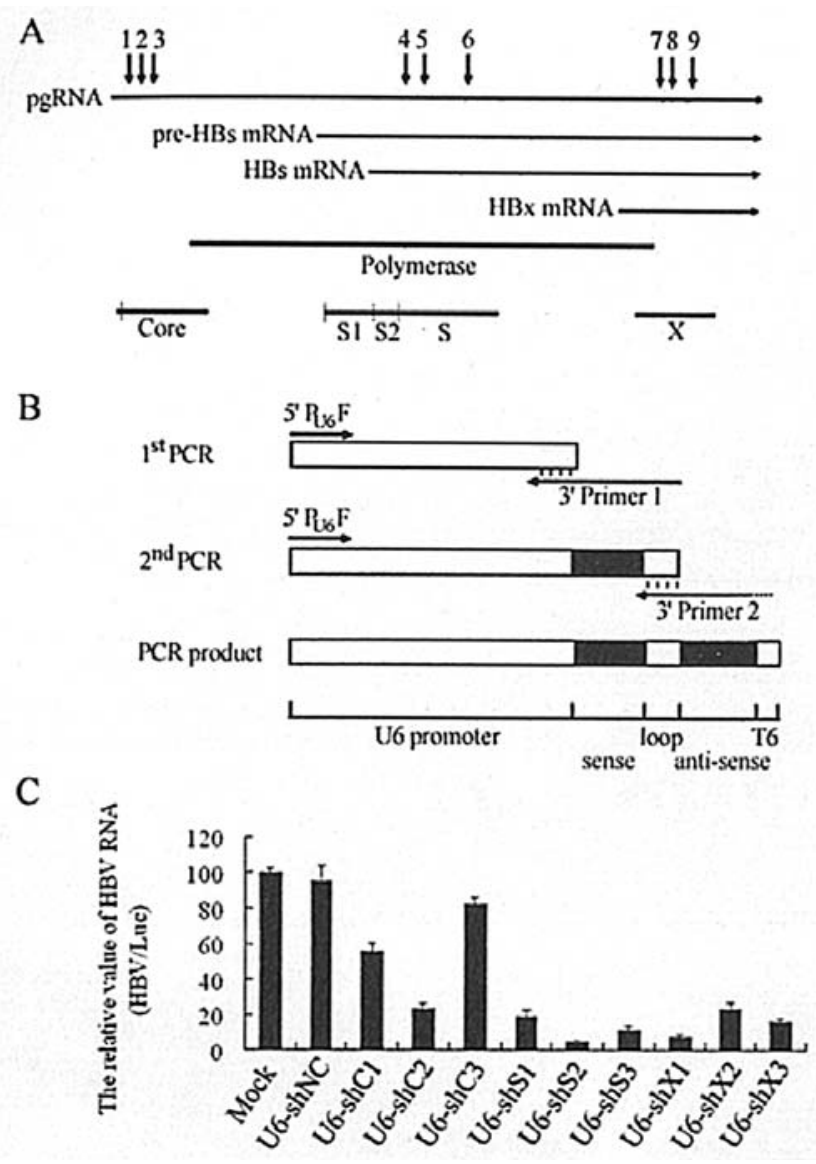

Figure 1. Screening for effective RNAi targeting sequences against HBV in the HepG2 cell line. (A) HBV RNA species and RNAi target sites. Downward arrows indicate the location of RNAi target sites within the four HBV transcripts $(1=\mathrm{C} 1,2=\mathrm{C} 2,3=\mathrm{C} 3,4=\mathrm{S} 1,5=\mathrm{S} 2,6=\mathrm{S} 3,7=\mathrm{X} 1,8=\mathrm{X} 2$; and $9=\mathrm{X} 3)$. The HBV open reading frames shown at the bottom row were aligned with $\mathrm{HBV}$ mRNAs. Core, $\mathrm{HBcAg}$; S1, large pre-surface antigen; S2, mid pre-surface antigen; S, HBsAg; and X, X gene. (B) Schematic diagrams of the overlap PCR to yield siRNA expression cassettes. (C) The reduction of the HBV RNA level by the U6-shDNA PCR products. The plasmid pHBV, pGL3-control and U6-shDNA PCR products were co-transfected into HepG2 cells. The levels of HBV RNA and luciferase mRNA were quantified by real-time PCR. The reduction in the ratio of HBV RNA to luciferase mRNA indicates the siRNA activity. The values shown are the average of three independent experiments (means \pm standard deviation).

the same forward primer $\mathrm{P}_{\mathrm{U} 6} \mathrm{~F}$ and a new reverse primer, $3^{\prime}$ Primer 2, which contained (5') poly (A) 6 , a 19-nt sense strand of siRNA, and a complementary sequence to the 9-nt loop (3') (Fig. 1B). The PCR products (U6-shDNA) were purified using a QIAquick PCR purification kit (Qiagen, Hilden, Germany).

Cell culture and transfection. HepG2 cells were maintained in DMEM supplemented with $10 \%$ fetal bovine serum at $37^{\circ} \mathrm{C}$. HepG2 cells were seeded at a density of $1.0 \times 10^{5}$ cells per well into 24 -well plates and grown to $\sim 80 \%$ at the time of transfection. Co-transfection of $0.1 \mu \mathrm{g}$ of luciferase expression plasmid pGL3-Control (Promega, WI, USA), $0.5 \mu \mathrm{g}$ of HBV replication competent plasmid pHBV (12) and $0.4 \mu \mathrm{g}$ of the PCR products was carried out with Lipofectamine 2000 (Invitrogen, MD, USA). Forty-eight hours posttransfection, cells were harvested and total RNA was isolated using an RNeasy kit (Qiagen) with DNase treatment. 
Generation of SiRNA expression HFV vectors and transduction. The construction of pGPSNI-GFP and helper $\mathrm{p} \Delta \mathrm{GP}$ have been described previously (13-15). The pHFVU6siS2 and pHFVU6-siX1 plasmids were constructed by replacing the SV40 promoter with the corresponding siRNA expression cassettes into the restriction enzyme sites of AccIII and SmaI on pGPSNI-GFP. To generate the dual siRNA expression plasmid pHFVU6-siSX, the AccIII site of plasmid pHFVU6-siX1 was blunted and the U6-shS2 fragment was cloned into this site (Fig. 2A). The control plasmid pHFVU6siNC was constructed with the random siRNA sequence as described previously. All constructs were confirmed by sequencing.

A series of HFVU6 vectors were produced by cotransfecting $293 \mathrm{~T}$ cells with vector plasmids and helper plasmid $\mathrm{p} \Delta \mathrm{GP}$. Supernatants were harvested 4 days posttransfection and purified by centrifugation at $5000 \mathrm{rpm}$ for $20 \mathrm{~min}$. The vectors were then concentrated by using the Amicon Ultra-15 centrifugal filters, $100 \mathrm{k}$ (Millipore, MA, USA). The titers of HFVU6 vectors were determined on $293 \mathrm{~T}$ cells. Four to five days after infection, cells were monitored and scored for GFP fluorescence under a fluorescence microscope (Olympus IX71). Transduction of 2.2.15 cells was carried out at a multiplicity of infection (MOI) of 30 as previously described (13).

ELISA assays and immunofluorescent staining. The levels of $\mathrm{HBsAg}$ and $\mathrm{HBeAg}$ in the media were determined using the AxSYM systems kit (Abbott, IL, USA) based on enzymelinked immunosorbent assay (ELISA) (16). All assays were performed three times independently.

The HBx protein was detected by immunofluorescent staining. Briefly, cells were washed and then fixed with $3 \%$ paraformaldehyde in phosphate-buffered saline (PBS) and permeabilized with $0.5 \%$ Triton $\mathrm{X}-100$ in PBS for $10 \mathrm{~min}$. After blocking with $3 \%$ bovine serum albumin (BSA) in PBS, cells were incubated with a monoclonal HBV X-protein antibody (Chemicon, CA, USA), followed by Cy3-conjugated secondary antibody (Sigma, MO, USA). Microscopic images were obtained with an Olympus fluorescence microscope.

Quantitative PCR analysis. Real-time PCR was performed to quantify HBV viral genomic DNA or RNA by using the HBV fluorescence quantitative PCR diagnostic kit (PG Biotech, Shenzhen, P.R. China). To quantify luciferase mRNA, primers 5'-GCGACCAACGCCTTAGATTGCAA-3', 5'GCGGTCAACGATGAAGAAGTG-3', and probe 5'-FAMATGGATGGCTACATTCTGGAGACATAG-3' were used. All reactions were performed in triplicate in 96-well optical reaction plates on an ABI PRISM 7900HT sequence detection system.

Semi-quantitative RT-PCR analysis of HBV transcripts. Total RNA was extracted from transduced cells using an RNeasy kit with DNase treatment. A first-strand synthesis reaction using ThermoScript reverse transcriptase (Invitrogen) was performed on $2 \mu \mathrm{g}$ of the RNA. PCR was carried out on $1 \mu \mathrm{l}$ of the first-strand reaction to identify HBV pgRNA (forward, 5'-CCTGGGTGGGTGTTAATTTGG-3'; reverse, 5'-GTAGTTTCCGGAAGTGTTGATAG-3'), HBs mRNA
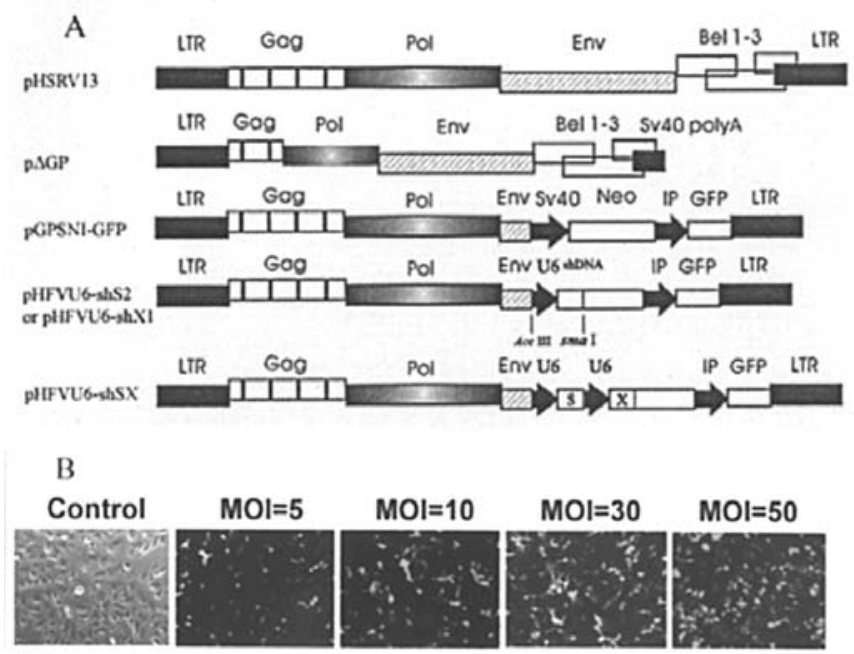

Figure 2. (A) Schematic representation of the HFV vectors. The provirus genome of HFV is shown in the top panel. All HFV vectors contain the EGFP gene expressed from the HFV internal promoter (IP) which helped to monitor transduction efficiency. (B) The transduction efficiency of HFV vectors at variant multiplicity of infection (MOI) in 2.2.15 cells. EGFP was detected and visualized using a fluorescence microscope (x200).

(forward, 5'-GCGGGGTTTTTCTTGTTGAC-3'; reverse, 5'CTACGAACCACTGAACAAAT-3'), HBx mRNA (forward, 5'-CCTGCGCGGGACGTCCTTTG-3'; reverse, 5'-CAGTC TTTGAAGTATGCCTC-3') and GAPDH mRNA (forward, 5'-GTAAAGTGGATATTGTTGCC-3'; reverse, 5'-TCAT ACTTCTCATGGTTCAC-3'). As a negative control for DNA contamination, PCR was also performed on the sample without conducting the RT step. Products were analyzed by electrophoresis on a $2 \%$ agarose gel.

Southern blot analysis. DNA was extracted with the QIAamp DNA mini kit (Qiagen), then digested and electrophoresed in $0.7 \%$ agarose gels and transferred to Hybond- $\mathrm{N}^{+}$membranes for Southern blot analysis. Full-length HBV DNA fragment was labeled with ${ }^{32} \mathrm{P}$ and used as the hybridization probe. Hybridization signals were analyzed using Phosphor-Imager 830 (Molecular Dynamics, Sunnyvale, CA).

\section{Results}

Screening for RNAi targeting sequences against $H B V$. Using the siRNA target finder program of Ambion, nine potential sequences against HBV were selected. After two-step overlap PCR as shown in Fig. 1B, nine corresponding PCR products containing human U6 promoter, sense, loop, antisense and terminator sequence were obtained. The PCR products were co-transfected with the pHBV and pGL3-control plasmids into HepG2 cells and real-time PCR was used to quantify the HBV RNA and luciferase mRNA. The reduction of HBV RNA by these PCR products was calculated after normalization with the control luciferase mRNA level. Our results showed that these PCR products targeting HBV viral genes reduced the HBV RNA quantity from 17.2-95.6\%, whereas the unrelated U6 PCR product (negative control NC) had no significant effect on the HBV RNA level ( $\mathrm{p}=0.126)$. Among these PCR products, U6-shS2 and U6-shX1 were the most 

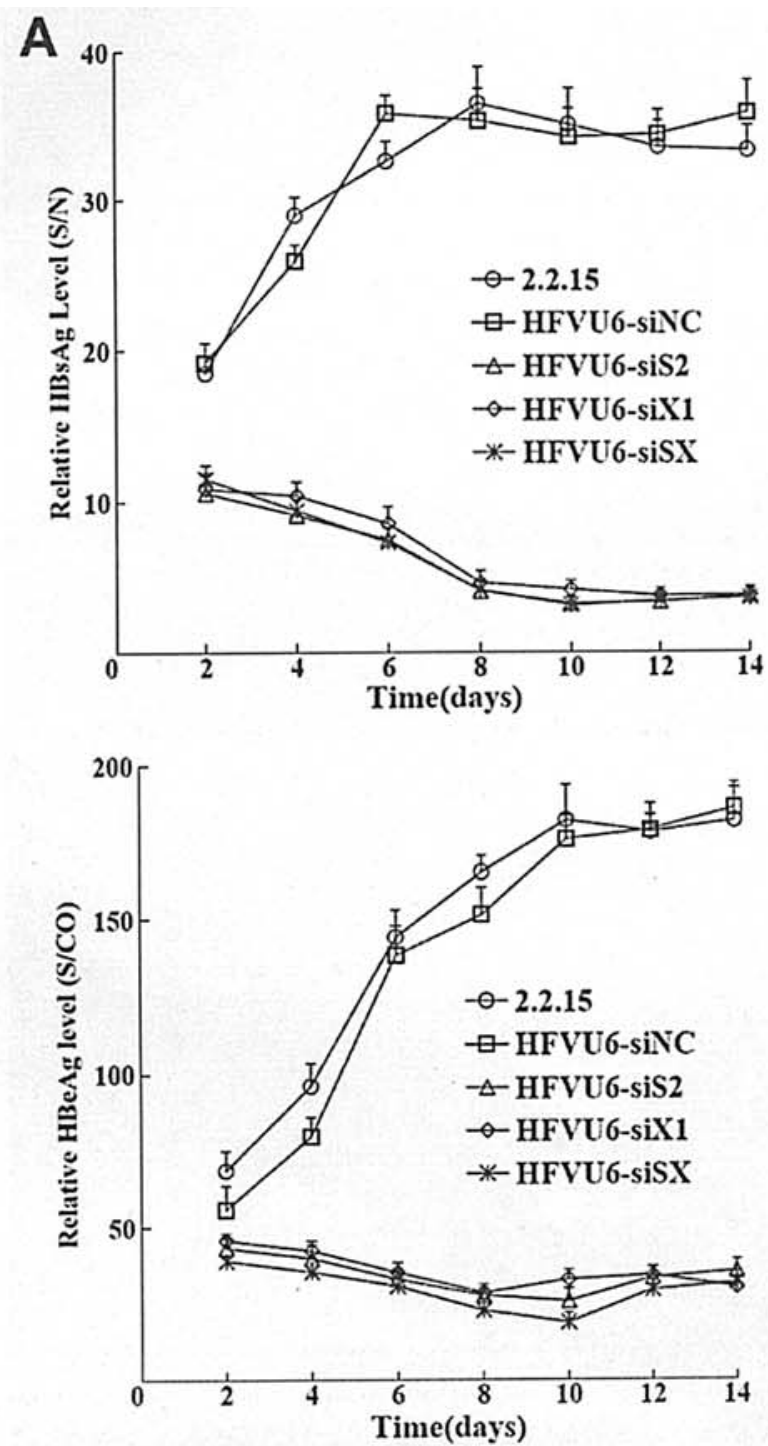

B

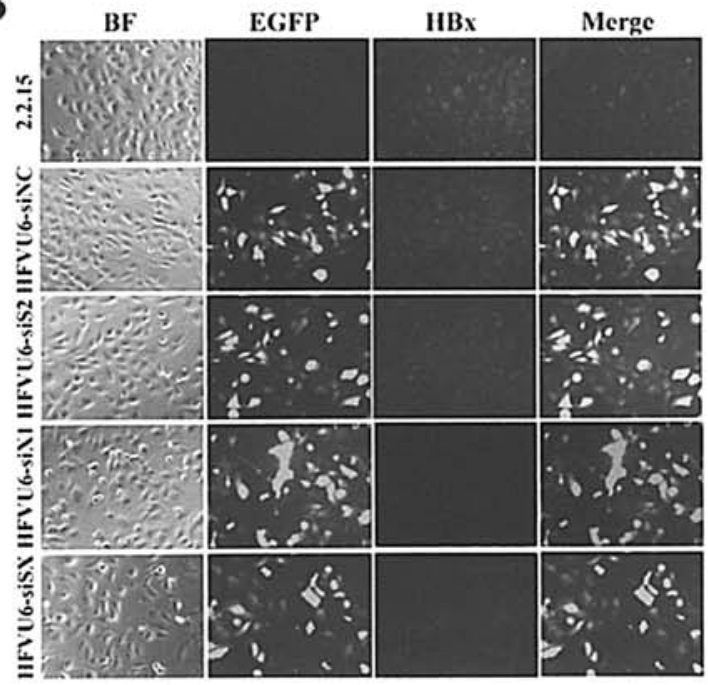

Figure 3. The effect of the HFV-based siRNAs on HBV viral gene expression in 2.2.15 cells. (A) The inhibitory effects of HFVU6-S2, HFVU6-X1 and HFVU6-SX on HBsAg and HBeAg expression in culture media. Normal 2.2 .15 cells and HFVU6-NC served as mock and negative controls. The values of $\mathrm{S} / \mathrm{N}$ and $\mathrm{S} / \mathrm{CO}$ represent the relative amounts of HBsAg and HBeAg, respectively. (B) Fourteen days post-transduction, the expression levels of HBx protein and EGFP were detected and visualized using a fluorescence microscope (x200); BF, bright field. effective and reduced HBV RNA by $95.6 \pm 3.51 \%$ and $92.3 \pm 1.52 \%$, compared to that of the mock control (Fig. 1C). Thus, U6-shS2 and U6-shX1 were selected to construct siRNA-expressing HFV vectors for further study.

Construction of siRNA expression HFV vectors and effective transduction. Two selected siRNA expression cassettes were placed into the HFV vector to generate the HFVU6-siS2, HFVU6-siX1 and a dual siRNA expression vector HFVU6siSX (Fig. 2A). The control plasmid pHFVU6-siNC was also constructed. All these HFV vectors contained the EGFP gene expressed from the HFV internal promoter (IP) which helped to monitor transduction efficiency. For determining the effective transduction titer of HFVU6 vectors, the 2.2.15 cells were transduced with the HFVU6-siNC vector at MOI of 5, 10, 30 and 50 for 7 days, and then the expression of EGFP was observed under an inverted fluorescence microscope. By counting the amount of cells with EGFP expression, it was shown that HFVU6-siNC transduced $30 \%$ cells at MOI of 5, 70\% at MOI of 10 and $95 \%$ at MOI of 30 and 50 (Fig. 2B). This same method was used to measure the titers of all the other HFVU6 vectors, and almost the same results were obtained (data not shown). Thus MOI of 30 was selected to transfect 2.2.15 cells for further study.

Inhibition of $H B V$ protein expression in 2.2 .15 cells with transduced HFV vectors expressing siRNA. 2.2.15 cells were infected with HFVU6-siS2, HFVU6-siX1, HFVU6-siSX and HFVU6-siNC at MOI of 30, respectively. The expression levels of HBsAg and $\mathrm{HBeAg}$ in culture media were determined with ELISA kits every 2 days post-transduction. As shown in Fig. 3A, three specific HFV siRNA vectors dramatically reduced the levels of $\mathrm{HBsAg}$ and $\mathrm{HBeAg}$ in the culture media, while the negative control vector had essentially no effect on HBV antigen expression. The reduction of HBsAg and $\mathrm{HBeAg}$ reached a peak on the 10th day post-transduction and remained level until day 14. HFVU6-siS2, HFVU6-siX1 and HFVU6-siSX reduced extracellular HBsAg and $\mathrm{HBeAg}$ levels by $90.5 \pm 1.4 \%, 83.6 \pm 0.7 \%, 92.4 \pm 2.7 \%$ and $84.8 \pm 1.7 \%$, $79.5 \pm 2.1 \%, 88.7 \pm 1.9 \%$, respectively, on the 10 th day posttransduction.

Immunofluorescence staining was employed to determine the expression levels of HBx protein in mock and transduced 2.2.15 cells on the 14th day post-transduction. In the cells transduced with HFVU6-siX1 or HFVU6-siSX, HBx signals were barely detectable, while in the cells transduced with HFVU6-siS2 or HFVU6-siNC, the signals from HBx were quite strong and almost no different from the HBx signals in the non-transduced cells. This indicated that HFVU6-siX1 and HFVU6-SX were able to efficiently inhibit the HBX protein expression while HFVU6-siS2 was ineffective (Fig. 3B, lane $\mathrm{HBx}$ ), demonstrating the expected specificity.

As in all the HFV siRNA expression vectors containing the EGFP expression cassette, the EGFP signals were able to be used as a marker to label the transduced cells. In this study, 14 days post-transduction, the expression of EGFP was observed in $>90 \%$ of the cells (Fig. 3B, lane EGFP). These data indicated that the HFV siRNA expression vectors infected the cells efficiently, and then induced stable and sequence-specific silencing of target genes of HBV. 


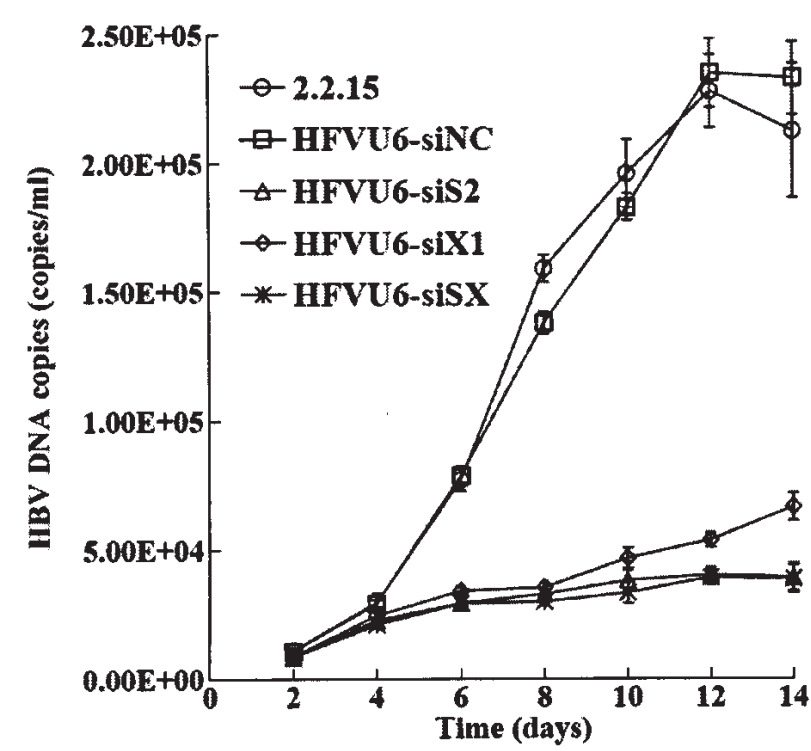

Figure 4. The inhibitory effects of HFVU6-S2, HFVU6-X1 and HFVU6-SX on HBV DNA in 2.2.15 cell culture media were measured by quantitative PCR. The values shown are the average of three independent experiments (means \pm standard deviation).

Inhibition of HBV DNA in 2.2.15 cell culture media. To determine the effect of siRNAs produced by foamy virus vectors on viral DNA replication, the HBV DNA in 2.2.15 cell culture media was quantified by real-time PCR analysis. Quantitative assay revealed that HBV DNA levels decreased 4 days post-transduction. The greatest reduction was observed at $82.33 \pm 2.17 \%, 76.4 \pm 1.68 \%$ and $83.96 \pm 1.32 \%$ $(\mathrm{p}<0.05)$ on day 12 for the three HFV siRNA vectors, respectively. Meanwhile, HBV DNA levels were not significantly changed between untreated 2.2.15 cells and the cells transduced with control vector HFVU6-siNC ( $p>0.05)$ (Fig. 4).

Long-term anti-HBV efficacy of siRNAs produced by foamy virus vector. To assess the long-term anti-HBV efficacy of siRNAs produced by foamy virus vectors, the transduced 2.2.15 cells were continually cultured. Three months posttransduction (about 30 passages), total RNA was isolated and analyzed by semi-quantitative RT-PCR (Fig. 5A). Different sets of primers for cDNA synthesis were used to determine the levels of pgRNA, HBs and HBx mRNA in transduced cells. Results indicated that the levels of pgRNA mRNA were significantly decreased by the infection of HFVU6siS2, HFVU6-siX1 and HFVU6-siSX, but not by HFVU6siNC. HBs mRNA was decreased more strongly by HFVU6siS2 and HFVU6-siSX, but slightly by HFVU6-siX1. Similarly, HBx mRNA was also decreased by HFVU6-siX1 and HFVU6-siSX. Notably, a reduced HBx mRNA level was observed in HFVU6-siS2 transduced cells. This was because pgRNA and HBs mRNAs also contained the HBx sequence at the $3^{\prime}$ end. As the primers used to amplify HBx recognized not only HBx mRNA but also pgRNA and HBs mRNA, the total RNA templates for the HBx PCR reaction were reduced when HFVU6-siS2 reduced the levels of pgRNA and HBs mRNAs. This resulted in the decrease of HBx PCR products shown on the agarose gel. This explanation was supported by
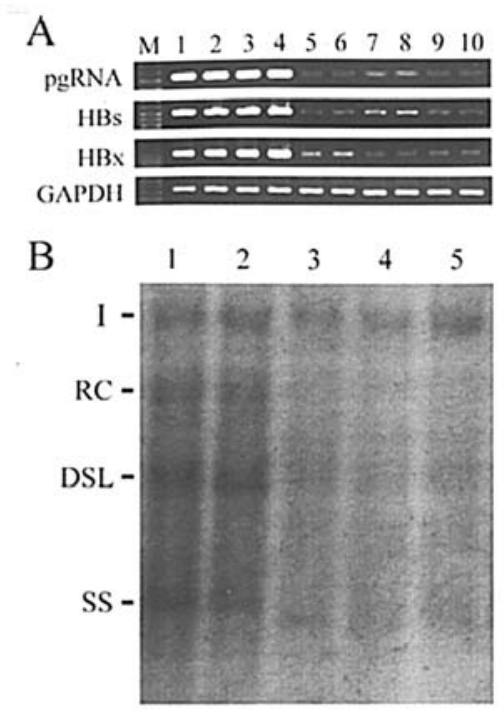

Figure 5. The effect of the HFV-based siRNAs on the HBV viral RNA and DNA in 2.2 .15 cells 3 months post-transduction. (A) The levels of HBV RNAs were detected in normal and transduced 2.2.15 cells by semiquantitative RT-PCR. GAPDH served as an internal control. (Lane 1 and 2) normal 2.2.15 cells; (3 and 4) 2.2.15 cells transduced with HFVU6-siNC; (5 and 6) 2.2.15 cells transduced with HFVU6-siS2; (7 and 8) 2.2.15 cells transduced with HFVU6-siX1; and (9 and 10) 2.2 .15 cells transduced with HFVU6-siSX. (B) Southern blot analysis of HBV DNA in 2.2.15 cells; (I) integrated HBV DNA as load-control of total cellular genomic DNA; (RC) relaxed circular DNA; (DSL) double-strand linear DNA; and (SS) single-strand DNA. (Lane 1) normal 2.2 .15 cells; (2) 2.2 .15 cells transduced with HFVU6-siNC; (3) 2.2 .15 cells transduced with HFVU6siS2; (4) 2.2.15 cells transduced with HFVU6-siX1; and (5) 2.2.15 cells transduced with HFVU6-siSX.

the previous immunofluorescence staining assay in which the HFVU6-siS2 had no effect on the HBx protein expression.

In addition, the inhibitory effects of the dual siRNA expression vector HFVU6-shSX on the levels of HBV pgRNA, HBs and HBx mRNA were greater than that of the single siRNA expression vector. This suggested that HFVU6siS2 and HFVU6-siX1 may cooperate to inhibit HBV mRNA expression.

To further determine the effect of the stably expressed siRNAs on HBV DNA replication, intracellular HBV DNA was isolated and analyzed by Southern blotting. The levels of relaxed circular (RC), double-strand linear (DSL) and singlestrand (SS) HBV DNA were clearly reduced in the cells transduced by HFVU6-siS2, HFVU6-siX1 and HFVU6-siSX but not in cells transduced by HFVU6-siNC. Density analysis further showed that HBV DNA replication intermediates were reduced by 76,68 and $83 \%$, respectively, compared to that of control (Fig. 5B).

\section{Discussion}

Recently, considerable attention has been directed toward the use of RNAi as a therapeutic method to treat a variety of diseases, especially viral diseases. Pioneer studies have demonstrated the possibilities of RNAi for treating serious viral diseases, including those caused by the human immunodeficiency virus, human corona virus, poliovirus, dengue virus, influenza virus and hepatitis $C$ virus (17-23). In addition, a number of studies have shown that RNAi reduces 
the expression of HBV genes or/and attenuates the replication of HBV genome in cell culture or in transgenic mice $(7,8,24-26)$. These studies have shed light on the development of new anti-HBV strategies.

To advance the application of RNAi in the inhibition of HBV, we employed a PCR-based RNAi strategy for a quick screening for effective anti-HBV siRNA sequences. A twostep overlap PCR strategy was utilized to generate a DNA template containing the U6 promoter and short hairpin DNA. The PCR products were directly co-transfected with a vectorbearing HBV genome into HepG2 cells, and HBV inhibition efficiencies were determined by real-time PCR. Compared to the traditional method, a multiple-step process which includes primer annealing, short hairpin sequence cloning into the RNAi expression vector, plasmid identification, amplification and transfection, this PCR-based strategy provides a rapid and easy assay for testing candidate siRNA sequences. By using this approach, we quickly identified two siRNA sequences (S2 and X1) having significant effects in the reduction of HBV RNAs.

Many studies have shown that viral vectors are good tools for effectively delivering foreign genes into cells. HFV is a member of the spumavirus subfamily of retroviruses (27). With its nonpathogenic nature, HFV has the advantage of being able to integrate into the host genome allowing for the stable expression of foreign genes, such as siRNAs. Therefore, in this study, HFV was employed to construct the siRNA expression vectors because it not only delivered the siRNA expression cassettes effectively but also allowed stable siRNA expression.

To study the long-term effects of selected siRNAs on HBV gene expression and viral replication, two single siRNA expression vectors HFVU6-siS2 and HFVU6-siX1, and a dual siRNA expression vector HFVU6-siSX were constructed and evaluated. These HFV siRNA vectors effectively inhibited multiple viral gene expression and viral DNA replication and most notably the repressions were stable for up to 3 months post-transduction. Several lines of evidence obtained from our study support the results. Firstly, ELISA and immunofluorescent staining experiments showed that viral proteins were significantly reduced in HFV siRNA vector transduced cells. Secondly, using RT-PCR, HBV RNAs were shown to be inhibited by these siRNA expression vectors. Finally, real-time PCR and Southern blotting showed that extracellular and intracellular HBV DNA were significantly reduced in HFV siRNA vector transduced cells.

Among the three vectors, HFVU6-siSX was a dual siRNA expression vector. It simultaneously expressed two siRNAs that targeted the $\mathrm{S}$ and $\mathrm{X}$ genes of HBV. Results from ELISA assays and semi-quantitated RT-PCR analysis consistently revealed that the dual siRNAs might have synergetic effects as HFVU6-siSX was more efficient on the inhibition of gene expression and viral protein production compared to that of the single siRNA expression vectors. In addition, the use of the multiple-siRNA expression vector may overcome the obstacle of siRNA resistance. The fact that a single nucleotide mutation allowed the virus to escape the effect of a single specific siRNA has been reported in poliovirus and HIV infections $(28,29)$. Recently, Wu et al also reported that a change of $\mathrm{T}$ to $\mathrm{C}$ at nucleotide 472 in an HBV clinical isolate resulted in siRNA resistance (26). Therefore, in this study we addressed such a potential problem by choosing targets in the relatively conserved viral sequences and by encoding two siRNAs that targeted different sites or genes simultaneously.

In conclusion, the PCR-based siRNA expression strategy provides a rapid and easy approach for testing candidate anti-HBV siRNA sequences and cloning selected siRNA expression cassettes into HFV vectors. RNAi based on the HFV vector achieved long-term inhibition of HBV gene expression and viral DNA replication in an HBV infection cell model. The strategy of constructing multi-siRNA expression vectors can enhance anti-HBV efficacy and overcome the evading mechanism of the virus.

\section{References}

1. WHO: Hepatitis B. Fact Sheet WHO/204. November, 1998.

2. Ayoola E, Balayab MS, Deinhardt F, Gust I, Kureshi AW and Maynard JE: Progress in the control of viral hepatitis: Memorandum from a WHO meeting. Bull WHO 66: 443-455, 1998.

3. Seeger C and Mason WS: Hepatitis B virus biology. Microbiol Mol Biol Rev 64: 51-68, 2000.

4. Custer B, Sullivan SD, Hazlet TK, Iloeje U, Veenstra DL and Kowdley KV: Global epidemiology of hepatitis B virus. J Clin Gastroenterol 38: S158-S168, 2004.

5. Fire A, Xu S, Montgomery MK, Kostas SA, Driver SE and Mello CC: Potent and specific genetic interference by doublestranded RNA in Caenorhabditis elegans. Nature 391: 806-811, 1998.

6. Arenz C and Schepers U: RNA interference: from an ancient mechanism to a state of the art therapeutic application? Naturwissenschaften 90: 345-359, 2003

7. Konishi M, Wu CH and Wu GY: Inhibition of HBV replication by siRNA in a stable HBV-producing cell line. Hepatology 38 : 842-850, 2003.

8. McCaffrey AP, Nakai H, Pandey K, Huang Z, Salazar FH, Xu H, Wieland SF, Marion PL and Kay MA: Inhibition of hepatitis B virus in mice by RNA interference. Nat Biotechnol 21: 639-644, 2003.

9. Paul CP, Good PD, Li SX, Kleihauer A, Rossi JJ and Engelke DR: Localized expression of small RNA inhibitors in human cells. Mol Ther 7: 237-247, 2003.

10. Castanotto D, Li H and Rossi JJ: Functional siRNA expression from transfected PCR products. RNA 8: 1454-1460, 2002.

11. Gou D, Jin N and Liu L: Gene silencing in mammalian cells by PCR-based short hairpin RNA. FEBS Lett 548: 113-118, 2003.

12. Fu WX, Kong Y and Wang Y: Regulation of HBV expression by core promoter and its upstream sequence. Chin J Virol 13: 215-223, 1997.

13. Li Z, Yang P, Liu $\mathrm{H}$ and Li WX: Construction of replicationdefective human foamy virus vector directing expression of foreign genes. Virol Sin 17: 114-118, 2002.

14. Zhang L, Li Z, Liu W, Liu H, He X and Li WX: Construction of replication-defective virus vector helper plasmid $\mathrm{p} \Delta \mathrm{GP}$ and cotransfection to HIC cell. J Wuhan Univ 49: 756-760, 2003.

15. Liu W, He X, Cao Z, Sheng J, Liu H, Li Z and Li W: Efficient therapeutic gene expression in cultured rat hippocampal neurons mediated by human foamy virus vectors: a potential for the treatment of neurological diseases. Intervirology 48: 329-335, 2005.

16. Taylor P, Pickard G, Gammie A and Atkins M: Comparison of the ADVIA Centaur and Abbott AxSYM immunoassay systems for a routine diagnostic virology laboratory. J Clin Virol 30 (suppl 1): 11-15, 2004.

17. Jacque JM, Triques K and Stevenson M: Modulation of HIV-1 replication by RNA interference. Nature 418: 435-438, 2002.

18. Lee NS, Dohjima T, Bauer G, Li H, Li MJ, Ehsani A, Salvaterra P and Rossi J: Expression of small interfering RNAs targeted against HIV-1 rev transcripts in human cells. Nat Biotechnol 20: 500-505, 2002.

19. He ML, Zheng B, Peng Y, Peiris JS, Poon LL, Yuen KY, Lin MC, Kung HF and Guan Y: Inhibition of SARS-associated coronavirus infection and replication by RNA interference. JAMA 290: 2665-2666, 2003. 
20. Coburn GA and Cullen BR: Potent and specific inhibition of human immunodeficiency virus type 1 replication by RNA interference. J Virol 76: 9225-9231, 2002.

21. Adelman ZN, Sanchez-Vargas I, Travanty EA, Carlson JO, Beaty BJ, Blair CD and Olson KE: RNA silencing of dengue virus type 2 replication in transformed C6/36 mosquito cells transcribing an inverted-repeat RNA derived from the virus genome. J Virol 76: 12925-12933, 2002.

22. Ge Q, McManus MT, Nguyen T, Shen CH, Sharp PA, Eisen HN and Chen J: RNA interference of influenza virus production by directly targeting mRNA for degradation and indirectly inhibiting all viral RNA transcription. Proc Natl Acad Sci USA 100: 2718-2723, 2003.

23. Kapadia SB, Brideau-Andersen A and Chisari FV: Interference of hepatitis C virus RNA replication by short interfering RNAs. Proc Natl Acad Sci USA 100: 2014-2018, 2003.

24. Giladi H, Ketzinel-Gilad M, Rivkin L, Felig Y, Nussbaum O and Galun E: Small interfering RNA inhibits hepatitis B virus replication in mice. Mol Ther 8: 769-776, 2003.
25. Moore MD, McGarvey MJ, Russell RA, Cullen BR and McClure MO: Stable inhibition of hepatitis B virus proteins by small interfering RNA expressed from viral vectors. J Gene Med 7: 918-925, 2005.

26. Wu HL, Huang LR, Huang CC, Lai HL, Liu CJ, Huang YT, Hsu YW, Lu CY, Chen DS and Chen PJ: RNA interferencemediated control of hepatitis B virus and emergence of resistant mutant. Gastroenterology 128: 708-716, 2005.

27. Flugel RM: Spumaviruses: a group of complex retroviruses. J Acquir Immune Defic Syndr 4: 739-750, 1991.

28. Gitlin L, Karelsky S and Andino R: Short interfering RNA confers intracellular antiviral immunity in human cells. Nature 418: 430-434, 2002.

29. Boden D, Pusch O, Lee F, Tucker L and Ramratnam B: Human immunodeficiency virus type 1 escape from RNA interference. $\mathrm{J}$ Virol 77: 11531-11535, 2003. 